\title{
CONTENT BASED MEDICAL IMAGE RETRIEVAL SYSTEM FOR ACCURATE DISEASE DIAGNOSES USING MODIFIED MULTI FEATURE FUSED XCEPTION MODEL
}

\author{
Mayank R. Kapadia \\ Ph.D. Scholar, Department of Electronics \& Communication, Uka Tarsadia University, \\ Surat, Gujarat-394350, India \\ kapadiamayank007@gmail.com \\ Dr. Chirag N. Paunwala \\ Professor, Sarvajanik College of Engineering and Technology, Surat \\ Surat, Gujarat- 395001, India \\ cpaunwala@gmail.com
}

\begin{abstract}
The Content Based Medical Image Retrieval (CBMIR) system helps to fulfill the need to diagnose the disease and provide proper treatment. The numerous complex deep neural networks are being applied to solve the same problem. This paper aims to design a simpler and accurate deep neural network-based CBMIR system. To achieve the same, the original Xception model is compressed by $58.33 \%$ by reducing the number of layers. The features of intermediate layers are fused to increase the accuracy of the proposed modified Xception model. This modified Multi-Feature Fused Xception (MFF-Xception) model is tested for two different X-ray image datasets: Image Retrieval in Medical Application (IRMA) dataset and the human's chest dataset. It gives an Average Precision Rate (APR) of $\mathbf{9 0 . 1 4 \%}$ for the IRMA dataset while providing $100 \%$ APR for the human chest dataset. It is also tested for another dataset containing skin images of seven different diseases and gives an APR of $100 \%$ for the skin dataset. The proposed modified MFF-Xception model also shows significant performance improvement over the original Xception model for all three datasets.
\end{abstract}

Keywords: Content Based Medical Image Retrieval (CBMIR); Xception model; Deep Learning.

\section{Introduction}

Content Based Medical Image Retrieval (CBMIR) retrieves medical images from the vast medical image dataset. CBMIR is the CBIR system's emerging field due to the wide range of image generation through the MRI, CT scan, X-ray, etc., via various clinics and hospitals. These medical images and their relevant cases create an excellent resource for clinical diagnoses and research study. The appropriate retrieved cases are useful for understanding the patient's critical illness and providing the correct treatment. The retrieved related cases can also help medical students to learn about the various disease and their diagnoses. It also allows researchers to invent new medicines for the specific disease from the past retrieved cases.

The medical imaging machine is a useful device to capture the low-level pictorial data from the patient. The doctor analyzes the high-level information and identifies the disease and its treatment from this low-level image data. The gap between these two, known as the semantic gap (Qayyum et al., 2017), is the major challenge in CBMIR. Many layers are available in the deep learning model. These layers will help to get the high-level information from this pictorial data. Hence, it will be used to reduce the semantic gap.

A considerable number of pre-trained models are available to extract the features from the input medical images. The pre-trained models are generally trained for the large dataset, and hence its weights and bias are not random. These pre-trained models are later on trained with no or few modifications for the required dataset. This technique is known as transfer learning. The modified Xception model is proposed in this paper to derive the necessary features from the medical images.

Further, the paper is arranged as follows. Section 2 illustrates the various related medical image retrieval techniques. The proposed modified Xception model is explained in section 3. The experimental analysis and 
discussion about the proposed model results are illustrated in section 4, and finally, the paper is ended with the conclusion in section 5 .

\section{Related Work}

The proposed work is to modify the pre-trained Xception model to reduce the number of layers and the number of parameters to train for the medical application. In this section, various research related to medical image retrieval has been explored.

In the initial stage of the CBMIR research, low-level feature extraction-based techniques were utilized to derive the medical images' features. In (Kimpan, Maneerat and Kimpan, 2018), Radial Inverse Force Histogram was used to retrieve the Diabetic eyes, which is useful for the retinal disorder diagnoses. Nikita et al. (Kashyap and Singh, 2017) has developed an HSV color histogram-based algorithm for the early detection and timely treatment of Diabetic Retinopathy. It has reduced the computation complexity, but the accuracy was also restricted. Some of the researchers have also used some classification techniques for image retrieval. The classification techniques can help to improve the image retrieval rate (Kapadia and Paunwala, 2018). The Speeded Up Robust Feature (SURF) technique provided comparatively good accuracy than the PCA and wavelet-based methods. The SVM classification technique has also provided comparatively good accuracy than the Relevance Vector Machine (RVM) and K-Nearest Neighbour (KNN) classification techniques. The combination of SURF and SVM also provided good accuracy for the various X-ray images (Lahari and Niranjana Krupa, 2018). M.Srinivas et al. (Srinivas et al., 2015) have used the dictionary learning method for the X-ray images. They have used mean and variance for the cluster's development. The Manifold Ranking (MR) technology was used for the optimization of precision and recall. It helped to develop a content-specific information graph that enhanced semantic relevance and allows for label uniformity. The relevance feedback mechanism was also introduced to support a learning-based system(Soundalgekar et al., 2018).

The classification-based technique's primary disadvantage is to provide handcrafted features from the image to the classification-based system. Hence, there is always a gap between human perception and handcrafted features. This gap is known as the semantic gap, which is the major challenge in the domain of the CBMIR. The foremost researchers have diverted towards machine learning-based technology due to advancements in processing technology like Graphical Processing Unit (GPU). The Convolution Neural Network (CNN) is the most popular model for 2D-images in machine learning.

The size of the medical image dataset is generally small, and CNN's training with this small dataset creates the problem of overfitting and convergence (Swati et al., 2019). Rafael S. Bressan et al. (Bressan et al., 2018) have proved that transfer learning can be utilized from a general context to a specific medical context. They have used the transfer learning method to retrieve the mammographic images. In (Xue et al., 2018), CNN has been used to develop a model from scratch and apply it to the spinal X-ray images for gender detection. They have also used the pre-trained DenseNet model for the same experiments, and the result was also improved with the transfer learning method. In (Kruthika, Rajeswari and Maheshappa, 2019), for the early detection of Alzheimer's disease, a 3D Capsule network (CapsNet) was proposed. This network was made up of the 3D-CNN and pre-trained 3Dautoencoder technology. This CapsNet model can learn fast and also handle the robustness effectively. The accuracy of this hybrid model was comparatively good than the Deep-CNN alone. In (Khatami et al., 2018), the two-level shrinking method using transfer learning and Radon transform has been used to overcome the problem of an imbalanced dataset and lack of big labeled data. The binary code was used with the transfer learning method for retinal image retrieval of diabetic retinopathy. It helped to increase image retrieval speed. It also helped to reduce the computation time and occupancy of memory (Wijesinghe, Gamage and Chitraranjan, 2019). For fast image retrieval, a hybrid model of CNN and Radon barcode was utilized in (Liu, Tizhoosh and Kofman, 2016)(Khatami et al., 2017), but this model needed some benchmark datasets for validation.

The transfer learning model can extract more valuable features from the images, improving the retrieval rate of the CBMIR system. Some researchers have tried to merge the features extracted from the multiple channels or the multiple transfer learning models to take advantage of the various models. Jinlong Hu et al. (Hu et al., 2019) have used a multi-channel 2-D CNN model for the retrieval of the MRI images. A small dataset has been selected for the experiment. In (Tian et al., 2016), the features were derived from the images through the different channels and then merged to represent the image's features. It helped to overcome the problem of the small dataset. It also improved the retrieval rate of the medical images. In (Guo and Yang, 2018), the ResNet model is utilized in multiple channels to derive the information from the skin lesions. The biographic information like age, gender may help to improve the accuracy further. In (Liu et al., 2019), the MR imaging data and demographic data were utilized to find clinical score regression. It was also used for the classification of brain disease using a deep multitask multi-channel learning method. Long D. Nguyen et al. (Nguyen et al., 2018) have used three pre-trained CNN models to derive features from the microscopic images. 
The concatenate model provided a better result than the single CNN model. The combination of low layer and high layer features can further improve the result. To identify the normal chest, pneumonia, and COVID- 19 from the X-ray images, two transfer learning models, Xception and ResNet50V2, were utilized to derive the features. The fusion of the features was used to identify the class of the query image. As the samples of COVID19 patients were small in quantity, the accuracy was tested for a small dataset (Rahimzadeh and Attar, 2020). This multi-channel feature extraction concept can extract the input medical image's various essential features to enhance retrieval. Hence, it reduces the semantic gap between the machine's visual information and the information analyzed by the doctor.

\section{Proposed Model}

In the proposed model, a transfer learning-based technique retrieves similar images from the medical image dataset. The Xception model (Chollet, 2017) is modified in the proposed transfer learning approach. This architecture has used a linear stack of depth wise separable convolution layers with residual connections. This architecture contains 36 convolution layers, which is divided into 14 modules. Except for the first and last modules, remaining all the modules have a linear residual connection around them. The architecture of the original Xception model is shown in Figure 1.

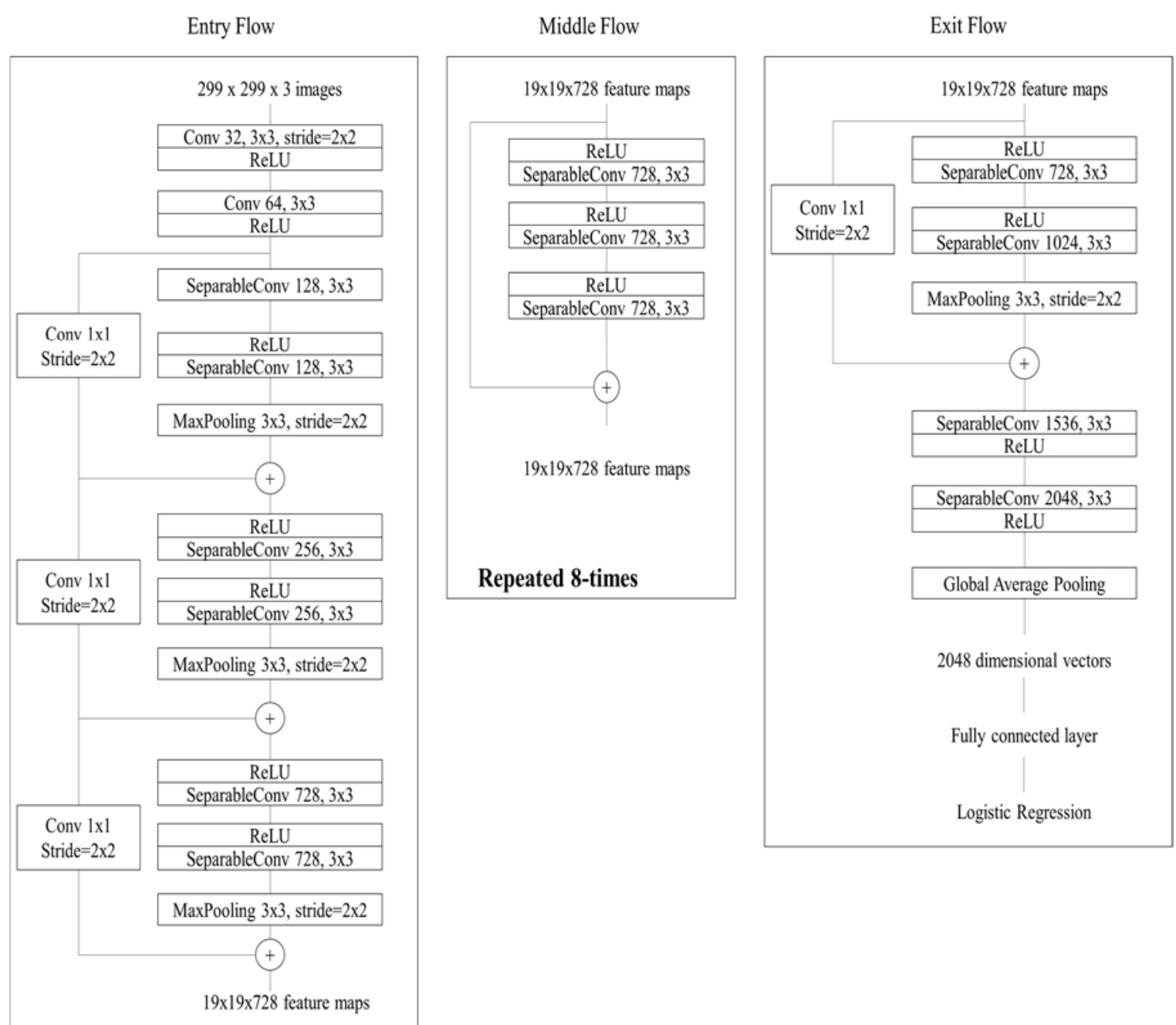

Fig. 1. The architecture of original Xception model (Chollet, 2017)

As shown in Figure 1, The Xception model has used the separable convolution instead of normal convolution. Let the input image is an RGB image, and its size is $14 \times 14 \times 3$. To get the same size of the output image, normal convolution and separable convolution take different multiplications. It is shown in Table 1 and Table 2. 
Table 1. The number of multiplications required in normal convolution

\begin{tabular}{|l|l|}
\hline \multicolumn{1}{|c|}{ Parameters } & \multicolumn{1}{c|}{ Normal Convolution } \\
\hline Input Image Size & $14 \times 14 \times 3$ \\
\hline Kernel Size & $3 \times 3 \times 3$ \\
\hline Total kernels & 256 \\
\hline $\begin{array}{l}\text { Number of times one kernel } \\
\text { moves over an input image }\end{array}$ & $12 \times 12$ \\
\hline Output Image Size & $12 \times 12 \times 256$ \\
\hline Total multiplications & $\begin{array}{l}=256 \times 3 \times 3 \times 3 \times 12 \times 12 \\
=9,95,328\end{array}$ \\
\hline
\end{tabular}

Table 2. The number of multiplications required in Separable convolution

\begin{tabular}{|l|l|l|}
\hline Parameters & (a) Depth wise convolution & (b) Pointwise convolution \\
\hline Input Image Size & $14 \times 14 \times 3$ & $12 \times 12 \times 3$ \\
\hline Kernel Size & $3 \times 3 \times 1$ & $1 \times 1 \times 3$ \\
\hline Total kernels & 3 & 256 \\
\hline $\begin{array}{l}\text { Number of times one kernel } \\
\text { moves over an input image }\end{array}$ & $12 \times 12$ & $12 \times 12$ \\
\hline Output Image Size & $12 \times 12 \times 3$ & $12 \times 12 \times 256$ \\
\hline Multiplications & $\begin{array}{l}=3 \times 3 \times 3 \times 12 \times 12 \\
=3888\end{array}$ & \\
\hline & $\begin{array}{l}=(\mathrm{a})+(\mathrm{b}) \\
=3888+1,10,592 \\
=1,14,480\end{array}$ & \\
\hline
\end{tabular}

So, from Table 1 and Table 2, the total multiplications required in separable convolutions are only 1,14,480, while for the same processes, the normal convolution required 9,95,328. Hence, the separable convolution is much faster than normal convolution. Therefore, the depth-wise separable convolution layers are used to reduce computational cost and memory requirements in the Xception model. Due to less computation, it will also take less time for the processing of the image.

In the original Xception model, the middle flow is repeated eight times, which means a total of three layers of separable convolution are repeated eight times, making 24 total separable convolution layers. As the medical dataset is small, more mathematical calculations can be reduced by reducing the number of layers in the middle flow. In our modified Xception model, this layer is repeated only a single time, which reduces the 21 separable convolution layers. Here, the entry flow is not modified because it was originally trained for a large ImageNet dataset. So, to take advantage of the transfer learning and at the same time to reduce the complexity, the middle flow is modified. The modified Xception model has reduced the number of separable convolution layers. The multi- channel feature concept is applied over here to benefit the input image's intermediate information to retrieve the medical images. The original and modified Xception model derives the 2048 features at the end of the exit. flow. The Multi-Feature Fused Xception (MFF-Xception) model fused some more features from the intermediate layers and generated 3504 features. The structure of the MFF-Xception model is shown in Figure 2.

Figure 2 shows that 728 intermediate features are extracted from the entry flow, more 728 features from the middle flow, and 2048 features from the exit flow are merged at the concatenate layer and make 3504 features for the image retrieval. Here, at the end of each flow, some useful features are derived, but in the original Xception model, these features were not considered for the classification. 


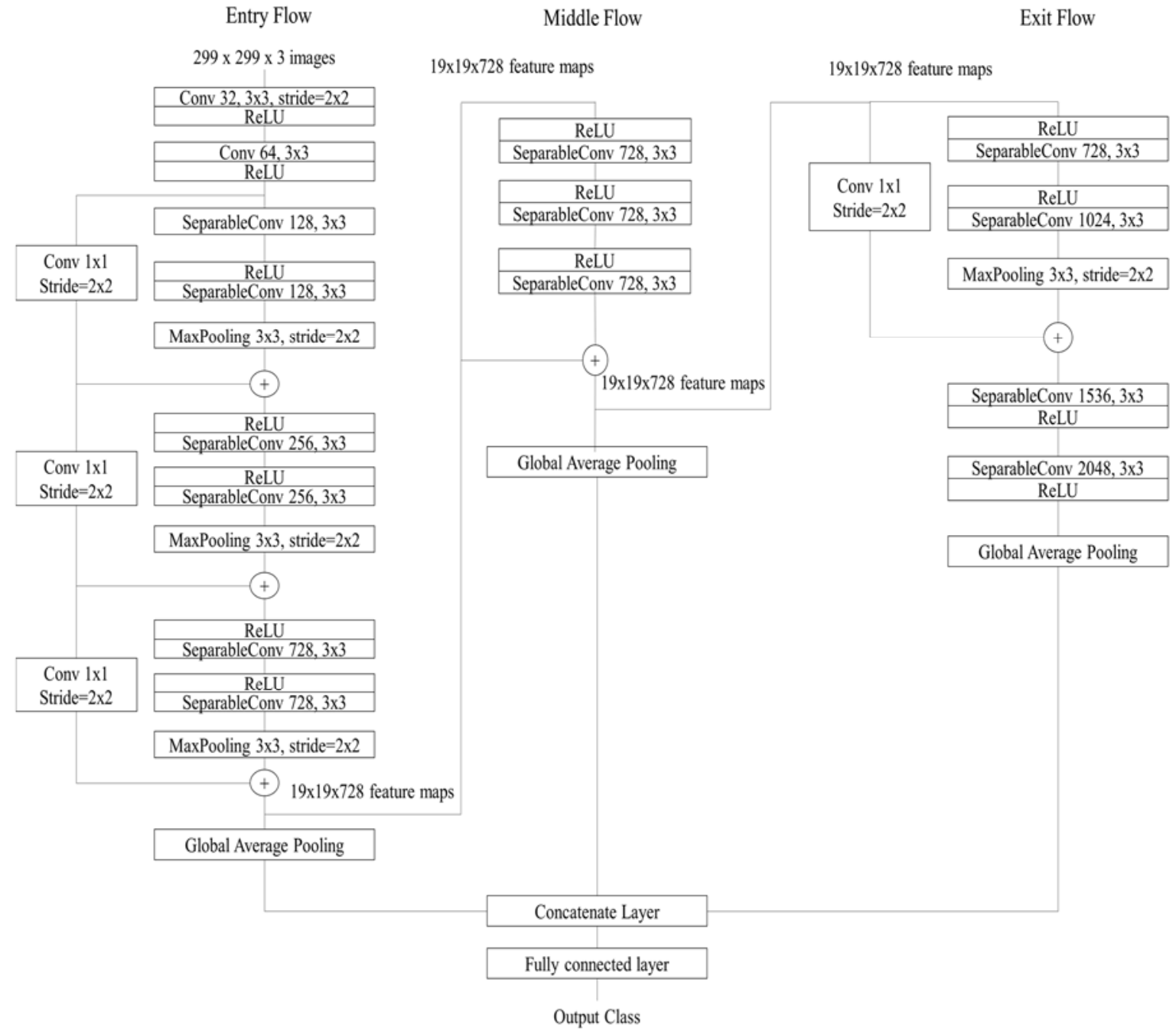

Fig. 2. The architecture of proposed Multi-Feature Fused Xception model (MFF-Xception)

In our modified model, these features were also added to impact the result. The MFF-Xception model will provide the class of the input query image. Based on the type, similar medical images will be retrieved from the dataset. The process of the retrieval of medical images using the MFF-Xception model is shown in Figure 3.

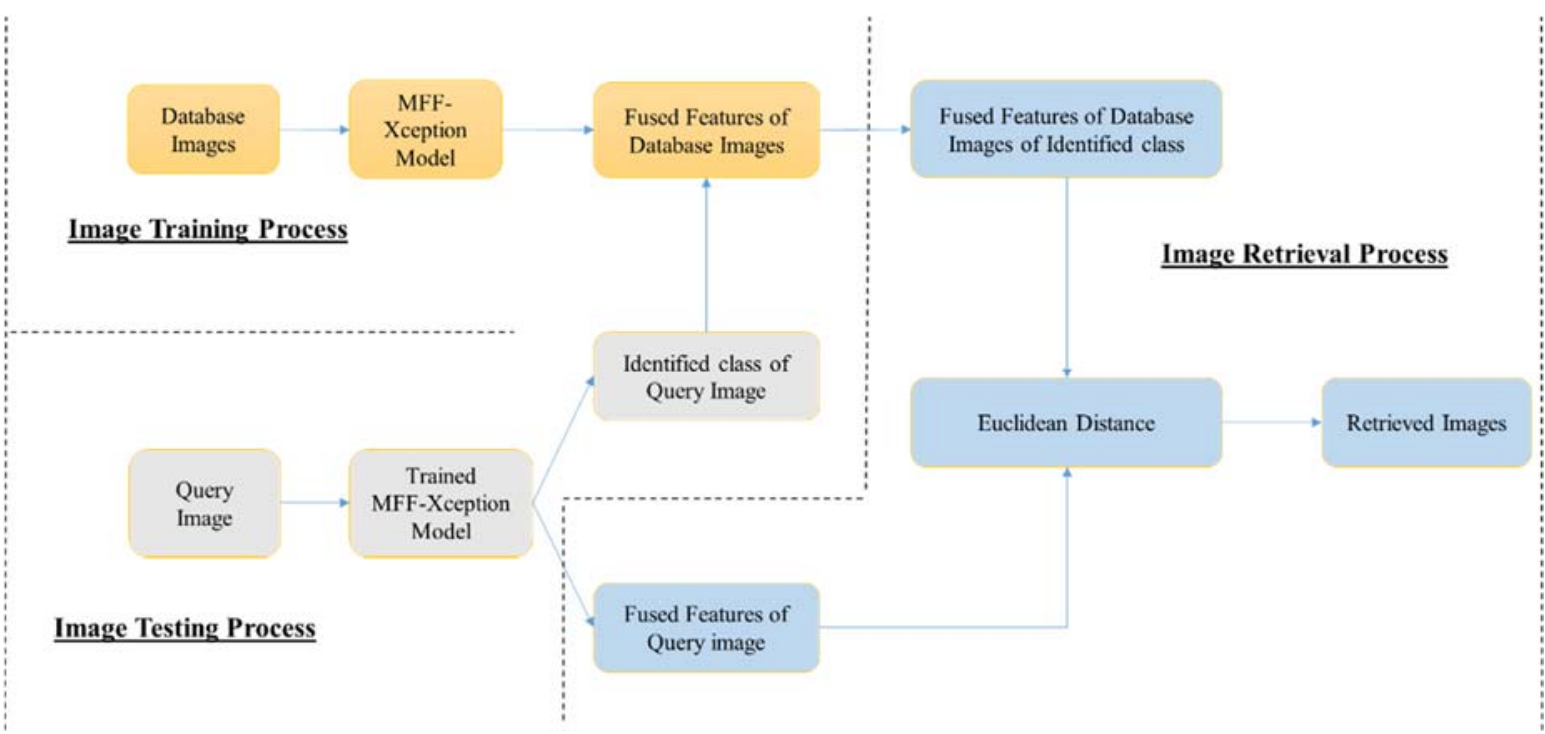

Fig. 3. Proposed MFF-Xception based CBMIR system 
The CBMIR system is divided into three-phase. During the image training process, the proposed MFFXception model is first learned through the medical image dataset. During the learning process, it modified the weights and biases in such a way to give the best retrieval results for the unknown medical images. It also stores the fused features of the database images for the image retrieval process. In the image testing process, the learned model will be validated through the unknown medical query images. The trained MFF-Xception model is used to identify the class of the query medical image. It also provides the fused features of the query image. During the third phase, the image retrieval process, the Euclidean distance between the fused features of the identified class's database images, and the query image's fused features are calculated using Eq. (1).

$$
E_{D}=\sqrt{\sum_{f=1}^{n}\left(M Q_{f}-M D_{f}\right)^{2}}
$$

Here, $E_{D}$ is the Euclidean distance between the query image's features and database images' features of the identified class. $\mathrm{MQ}_{\mathrm{f}}$ is a feature vector of the medical query image, and $\mathrm{MD}_{\mathrm{f}}$ is a feature vector of the identified class medical dataset image, and $\mathrm{f}$ is the number of features. The images are arranged in ascending order based on calculated Euclidean distance. Hence, most similar images will be displayed first, and most dissimilar images will be shown last.

The accuracy parameter is used to measure how accurately the MFF-Xception model recognizes the provided query medical image class. It is defined as the ratio of total correct prediction to the total prediction. The precision rate is defined as the total relevant images retrieved from the medical image dataset to the total images retrieved. It is given by Eq. (2).

$$
P R_{M}=\frac{\text { Total relevant images retrived }}{\text { Total images retrived }}
$$

Here, $\mathrm{PR}_{\mathrm{M}}$ is the precision rate of the $\mathrm{M}^{\text {th }}$ medical query image. The Average Precision Rate (APR) is used to measure the performance of the CBMIR system.

$$
A P R=\frac{1}{|N|} \sum_{j=1}^{N} P R_{j}
$$

Here, $\mathrm{N}$ is the total number of medical query images. The average of all precision rate of medical query images is known as Average Precision Rate (APR), shown in Eq. (3). In our analysis, when the image class is perfectly-recognized, similar images are retrieved from the matching image class. Hence, the APR gives similar outcomes as classification accuracy.

\section{Results and Discussion}

The experiment has been done on the Google colaboratory platform. This platform provides NVIDIA K80 GPU with 12GB of memory. The proposed MFF-Xception model is verified for three different medical image datasets. Python is the programming language used for these experiments. The proposed MFF-Xception model is developed using the KERAS framework. As a backend, the KERAS framework uses TensorFlow. The APR is utilized to evaluate the performance of the proposed model. The proposed MFF-Xception model has been evaluated for the Image Retrieval Medical Application (IRMA) dataset (Lahari and Niranjana Krupa, 2018)(Srinivas et al., 2015)(Soundalgekar et al., 2018)(Liu, Tizhoosh and Kofman, 2016) (Khatami et al., 2017), which is the standard medical dataset for the image retrieval system. This dataset has been collected from the Dr. Thomas Deserno, Department of Medical Informatics, Aachen University of Technology, Germany. The proposed MFF-Xception model has been evaluated for the $2500 \mathrm{x}$-ray images. These images are classified into nine different classes. The class of the images is decided based on the parts of the human body. The names of these various classes are 'Head', 'Mouth', 'Neck', 'Chest', 'Spine', 'Abdomen', 'Upper extremity', 'Hip', and 'Leg'. Figure 4 shows images of these mentioned classes. 

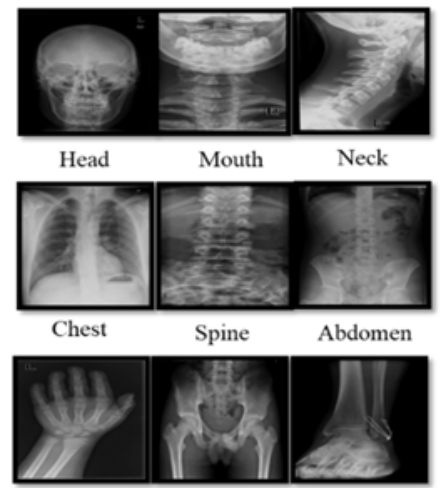

Upper Extremity Hip Leg

Fig. 4. Sample images of IRMA dataset

The proposed MFF-Xception model is also evaluated for two different datasets. These two datasets are collected from Dr. Milind Parekh, a Homeopathic practitioner. One of the datasets contains X-ray images of the human chest. This dataset has 1090 images, and the size of each image is $512 \times 512$. This dataset is categorized into five different classes based on chest disease. The classes' names based on the disease are 'Normal chest', 'Pleural effusion', 'Tuberculosis', 'Viral Pneumonia', and 'COVID'. Figure 5 shows images of the human chest dataset.

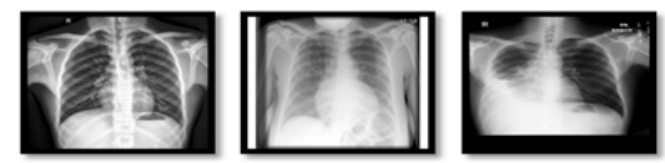

Normal Chest Pleural effusion Tuberculosis

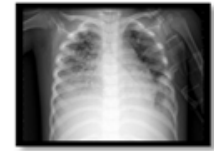

Viral Pneumonia

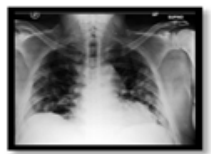

COVID

Fig. 5. Sample images of human chest disease dataset

Another dataset contains skin disease-related images. This dataset has 2050 images, and the size of each image is $512 \times 512$. This dataset is categorized into seven different classes based on various skin disease. The names of the various disease classes are 'vitiligo', 'psoriasis', 'urticaria', 'ringworm', 'lichen planus', 'Wart', and 'acne'. Figure 6 shows images of the skin disease dataset.
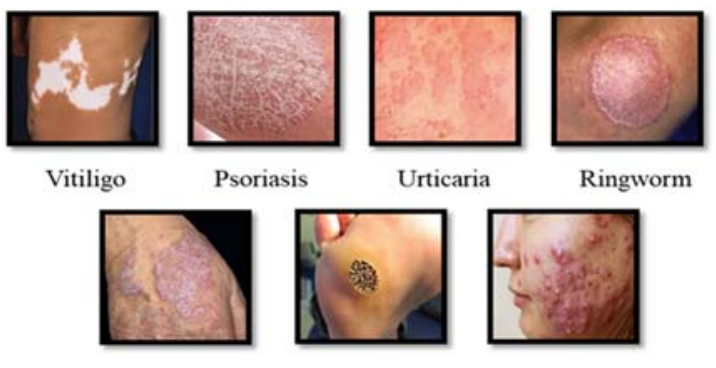

Ringworm

Lichen planus

Wart

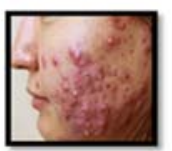

Acne

Fig. 6. Sample images of skin disease dataset

The dataset is distributed among the training set, validation set, and testing set for the evaluation. The ratio of distribution is 60:20:20, respectively. This is applicable to all three datasets. The pre-trained models are trained using transfer learning techniques. The transfer learning technique is mainly designed to utilize the same network for various applications. The learning parameters like wights and biases are random in the model developing from scratch. These learning parameters are not random in the transfer learning case because they have already learned some characteristics from the previous training. Due to this non-randomness characteristic of the learning parameters, the model learned fast for the new datasets and saves time for the training. In this transfer learning model, the last few layers will be trained through the new dataset while the earlier layers are frozen. The final three layers are utilized for the training in this experiment. After the training of the transfer learning model, it will 
be evaluated with the non-trained testing dataset. The result of these testing datasets is compared with the actual ground truth to validate the model's performance. The benchmark pretrained models are evaluated for the two xray medical image datasets. It is shown in Figure 7.

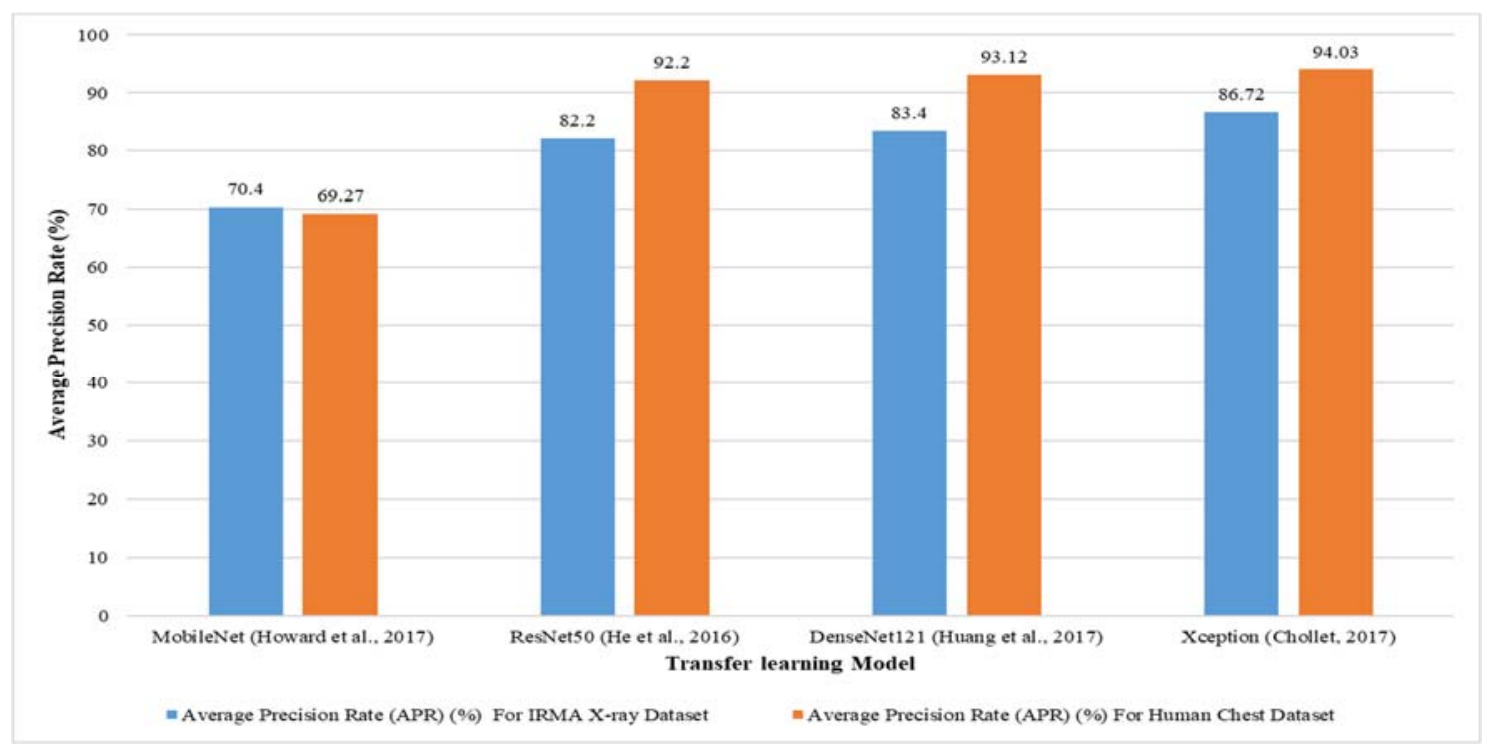

Fig. 7. Comparative analysis of various transfer learning models

As shown in Figure 7, the Xception model (Chollet, 2017) provides comparatively good results than MobileNet (Howard et al., 2017), the ResNet50(He et al., 2016), and DenseNet (Huang et al., 2017) transfer learning based models for both IRMA and human chest datasets. Due to this reason, the Xception model is modified for the proposed model. In the modified Xception model, the 8- times repetition has been removed, which removes the number of convolution layers and reduces the mathematical operation. It will also help to reduce the number of learning parameters and, thus, training time. Table 3 shows how the modified Xception model reduces the number of convolution layers and the number of learning parameters.

Table 3. Xception model vs. Modified Xception model

\begin{tabular}{|l|c|c|}
\hline \multicolumn{1}{|c|}{ Model } & $\begin{array}{c}\text { Number of Convolution } \\
\text { Layers }\end{array}$ & $\begin{array}{c}\text { Number of Learning } \\
\text { Parameters }\end{array}$ \\
\hline Xception Model (Chollet, 2017) & 36 & $20.87 \mathrm{M}$ \\
\hline Modified Xception Model & 15 & $9.55 \mathrm{M}$ \\
\hline
\end{tabular}

As per Table 3, the original Xception model is compressed by $58.33 \%$ for the medical image datasets. Also, learning parameters are compressed by $54.24 \%$. This modified Xception model is also evaluated for all three image datasets. The results for the modified Xception model for all three datasets are shown in Table 4.

Table 4. The performance of modified Xception model

\begin{tabular}{|l|c|c|c|}
\hline \multicolumn{1}{|c|}{ Dataset } & IRMA Dataset & $\begin{array}{c}\text { Human Chest } \\
\text { Dataset }\end{array}$ & $\begin{array}{c}\text { Skin Disease } \\
\text { Dataset }\end{array}$ \\
\hline Training Accuracy (\%) & 99.87 & 100 & 99.59 \\
\hline Validation Accuracy (\%) & 89.46 & 98.62 & 100 \\
\hline Testing Accuracy (\%) & 89.74 & 95.87 & 99.76 \\
\hline
\end{tabular}

These results have been compared with the original Xception model for testing images of all three datasets. Figure 8 shows the comparison of the modified Xception model with the original Xception model (Chollet, 2017). 


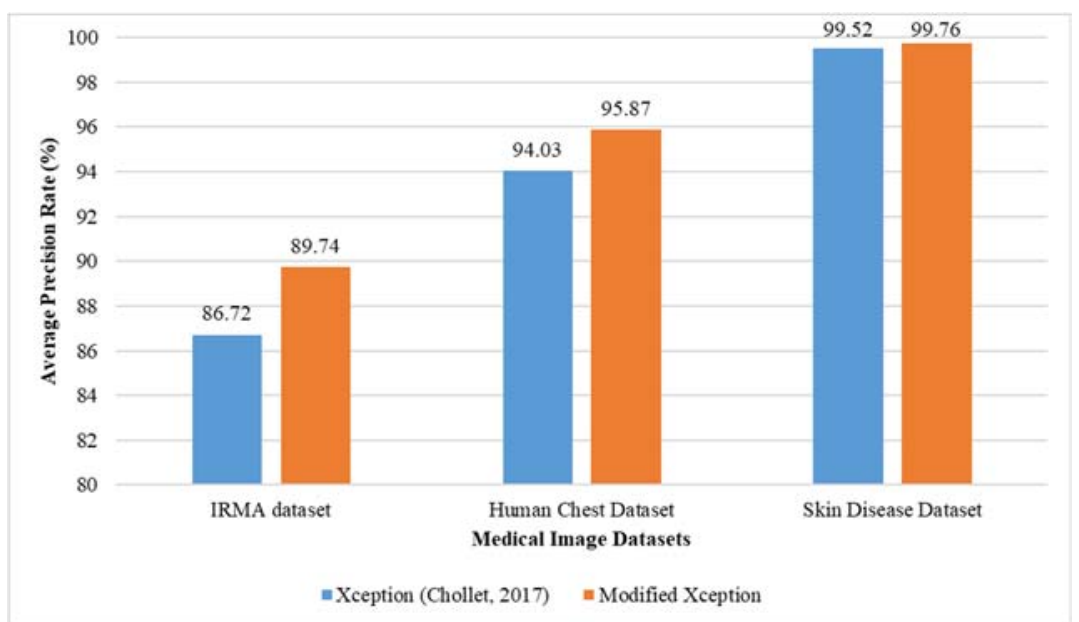

Fig. 8. Comparison of APR for original and modified Xception model

As far as the small dataset concern, fewer convolution layers are required to extract the high-level features to reduce the semantic gap (Kapadia, 2018). As shown in Figure 8, even the number of layers reduced by $58.33 \%$ from the original Xception model, the accuracy is improved. Thus, the smaller datasets required a lesser number of layers. As the number of layers is reduced, a lesser number of learning parameters will be utilized. Lesser learning parameters will occupy less memory. Hence, a modified Xception model improves the accuracy of the medical datasets with a reduced number of learning parameters, reduced training time, and lesser memory occupancy.

Further, the accuracy can be improved by adding the concept of multi-feature fusion. The multiple features of intermediate layers are fused to take the benefits of the information derived from the intermediate layers. It is shown in Figure 2. These intermediate features are combined with the features of the modified Xception model through the concatenation layer. The results for the MFF-Xception model for all three datasets are shown in Table 5.

Table 5. The performance of MFF-Xception model

\begin{tabular}{|l|c|c|c|}
\hline \multicolumn{1}{|c|}{ Dataset } & IRMA Dataset & $\begin{array}{c}\text { Human Chest } \\
\text { Dataset }\end{array}$ & $\begin{array}{c}\text { Skin Disease } \\
\text { Dataset }\end{array}$ \\
\hline Training Accuracy (\%) & 99.73 & 100 & 99.67 \\
\hline Validation Accuracy (\%) & 92.25 & 100 & 100 \\
\hline Testing Accuracy (\%) & 90.14 & 100 & 100 \\
\hline
\end{tabular}

These results have been compared with the original Xception model and modified Xception model for testing images of all three datasets. Figure 9 shows the MFF-Xception model's comparison with the modified Xception model and the original Xception model (Chollet, 2017).

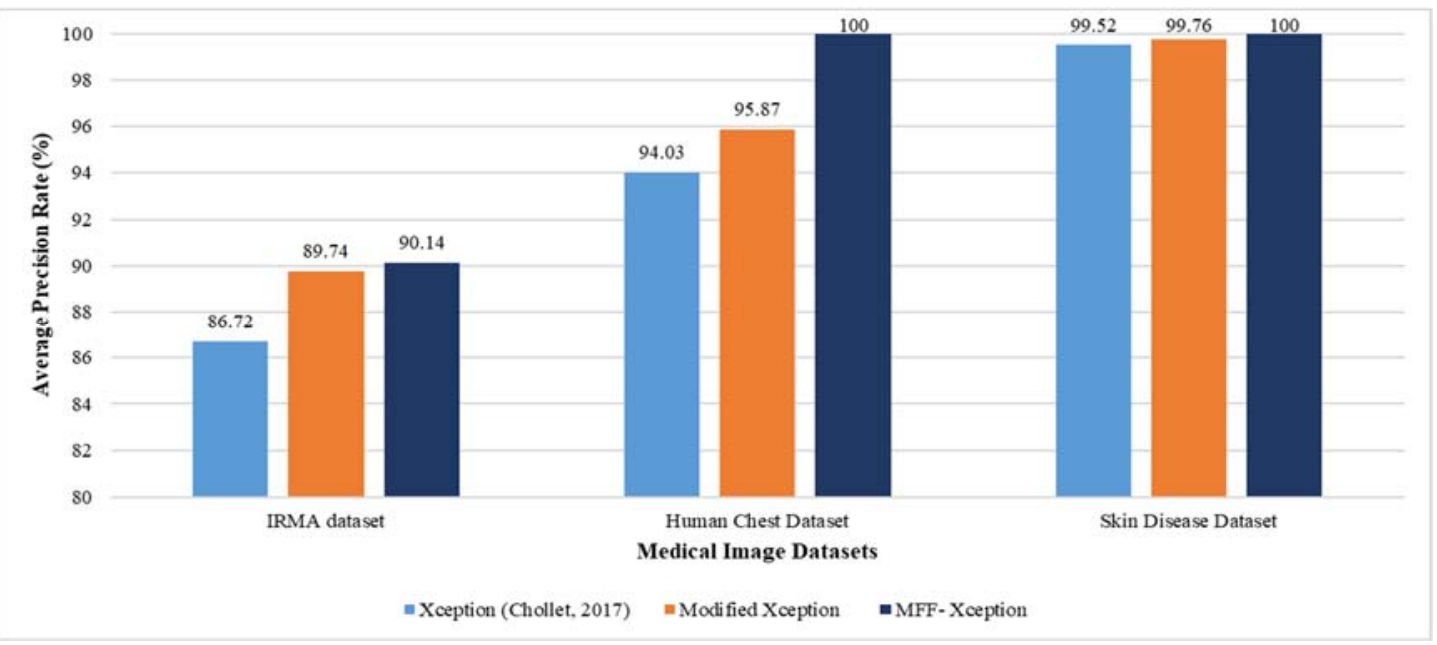

Fig. 9. Comparison of APR for Xception, modified Xception and MFF-Xception model

As shown in Figure 9, the MFF-Xception model provides 90.14\% accuracy for the IRMA dataset, $100 \%$ accuracy for the chest disease dataset, and 100\% accuracy for the skin disease dataset. The MFF-Xception model 
improves the results by increasing the features by $72 \%$ than the original Xception model. Also, the MFF-Xception model gives the best outcome for all three datasets compare to the original and modified Xception model.

The result of the proposed MFF-Xception model is also compared with the M2D CNN model (Hu et al., 2019). The M2D-CNN model has also fused the features from the three different channels. Each channel has used the two layers of CNN. Table 6 shows the comparison of the proposed MFF-Xception model's result with the result of the M2D-CNN model.

Table 6. Comparison of M2D-CNN model (Hu et al., 2019) and proposed MFF-Xception model

\begin{tabular}{|c|c|c|}
\hline \multirow{2}{*}{ Dataset } & \multicolumn{2}{|c|}{ Testing Accuracy (\%) } \\
\cline { 2 - 3 } & M2D-CNN (Hu et al., 2019) & Proposed MFF-Xception model \\
\hline IRMA & 84.9 & 90.14 \\
\hline Human Chest & 93.11 & 100 \\
\hline Skin Disease & 98.12 & 100 \\
\hline
\end{tabular}

As shown in Table 6, the proposed MFF-Xception model performs exceptionally well compared to the M2D CNN (Hu et al., 2019) model. For the IRMA dataset, the result is improved by $5.81 \%$, while for the human chest dataset, the result is improved by $6.89 \%$, and for the skin dataset, the result is improved by $1.88 \%$.

The retrieval results of the CBMIR system using the proposed MFF-Xception model is shown in Figure 10. The images are retrieved for the IRMA dataset. The x-ray image of the 'leg' is provided as an input to the proposed MFF-Xception model. The model retrieved similar types of medical images from the 'leg' class. It is shown in Figure 10 (a) and Figure 10 (b), respectively.

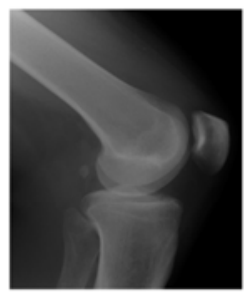

(a)

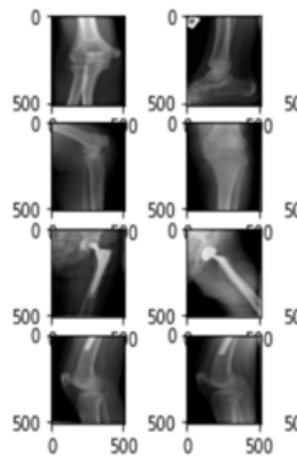

(b)

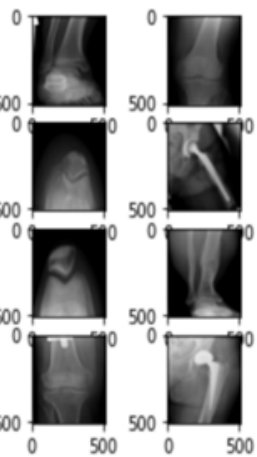

Fig. 10. (a) Query Image for 'Leg' (b) Retrieved images of 'Leg'

As shown in Figure 11 (a), the X-ray image of a human chest for "Viral Pneumonia' disease is given as a query image to the proposed MFF-Xception model. The proposed model has identified the class of the medical query image and based on that, it has retrieved related images of the 'Viral Pneumonia' class, as shown in Figure 11 (b).

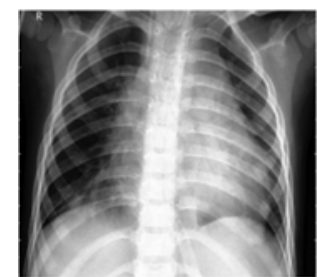

(a)

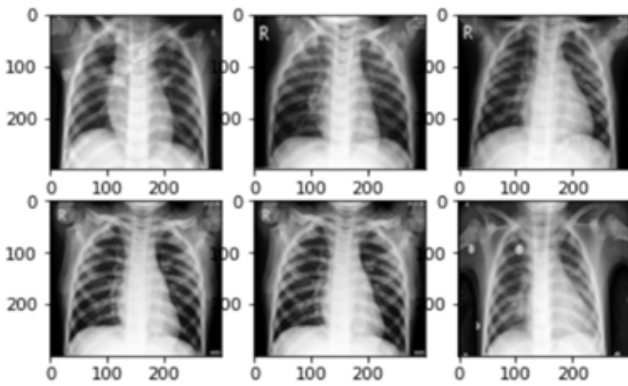

(b)

Fig. 11. (a) Query Image for 'Viral Pneumonia' (b) Retrieved images of 'Viral Pneumonia'

Similarly, the MFF-Xception model is also evaluated for the skin dataset. The model has retrieved similar images for the 'urticaria' skin disease query image. It is shown in Figure 12 (a) and Figure 12 (b), respectively. 


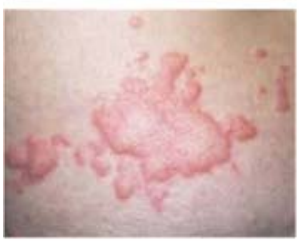

(a)
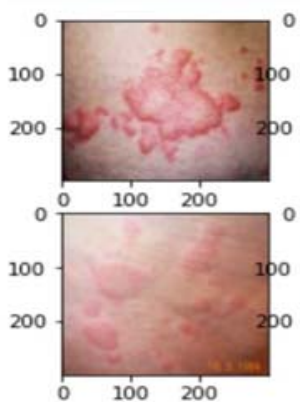

100200

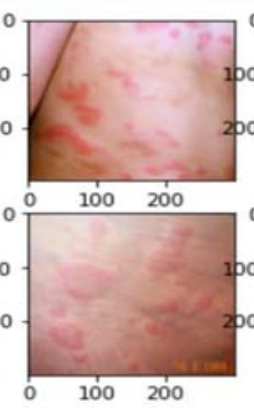

(b)

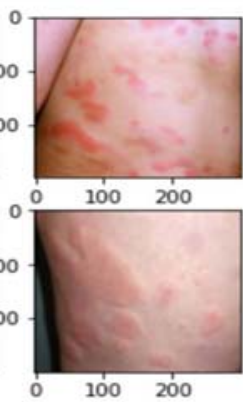

200

Fig. 12. (a) Query Image for 'Urticaria' (b) Retrieved images of 'Urticaria'

These retrieved similar medical images can also help to recover the treatment given to those specific patients. The previous treatment's effectiveness can help the doctor to decide the proper treatment of the current patient.

\section{Conclusion}

The Xception model is modified to reduce the number of trainable parameters and convolution layers without affecting the model's performance. It helps to improve the speed of the operation by decreasing the number of the convolution operation. The reduction in the number of trainable parameters helps to save memory storage. The multi-feature fusion technology is introduced in the Modified Xception model to benefit the intermediate layer's features. Due to this fusion model, the last layer has many more meaningful features than the original model. It helps to improve the performance of image retrieval. These more features helped to reduce the semantic gap. The experimental results show that the proposed MFF-Xception model's performance is comparatively better than the original Xception and modified Xception model. The results are also better than the literature's multi-channel feature fusion-based technology. However, the experiment has been done on a limited class of images. Hence, increasing the number of classes may also lead to better performance.

\section{Acknowledgments}

The Authors would like to acknowledge Dr. Thomas Deserno, Department of Medical Informatics, Aachen University of Technology, Germany for providing dataset to do this research work. The authors also would like to express sincere gratitude to Dr. Milind Parekh for his guidance throughout this research.

\section{References}

[1] Bressan, R. S. et al. (2018) 'DOCToR: The Role of Deep Features in Content-Based Mammographic Image Retrieval', Proceedings IEEE Symposium on Computer-Based Medical Systems. IEEE, 2018-June, pp. 158-163. doi: 10.1109/CBMS.2018.00035.

[2] Chollet, F. (2017) 'Xception: Deep learning with depthwise separable convolutions', Proceedings - 30th IEEE Conference on Computer Vision and Pattern Recognition, CVPR 2017, 2017-Janua, pp. 1800-1807. doi: 10.1109/CVPR.2017.195.

[3] Guo, S. and Yang, Z. (2018) 'Multi-Channel-ResNet: An integration framework towards skin lesion analysis', Informatics in Medicine Unlocked. Elsevier Ltd, 12(June), pp. 67-74. doi: 10.1016/j.imu.2018.06.006.

[4] He, K. et al. (2016) 'Deep residual learning for image recognition', Proceedings of the IEEE Computer Society Conference on Computer Vision and Pattern Recognition, 2016-Decem, pp. 770-778. doi: 10.1109/CVPR.2016.90.

[5] Howard, A. G. et al. (2017) 'MobileNets: Efficient Convolutional Neural Networks for Mobile Vision Applications'. Available at: http://arxiv.org/abs/1704.04861.

[6] Hu, J. et al. (2019) 'A Multichannel 2D Convolutional Neural Network Model for Task-Evoked fMRI Data Classification', Computational Intelligence and Neuroscience, 2019(i). doi: 10.1155/2019/5065214.

[7] Huang, G. et al. (2017) 'Densely connected convolutional networks', Proceedings - 30th IEEE Conference on Computer Vision and Pattern Recognition, CVPR 2017, 2017-Janua, pp. 2261-2269. doi: 10.1109/CVPR.2017.243.

[8] Kapadia, M. R. and Paunwala, C. N. (2018) 'Improved CBIR system using Multilayer CNN', 2018 International Conference on Inventive Research in Computing Applications (ICIRCA) pp 840\{845, DOI 10.1109/ICIRCA.2018.8597199.

[9] Kapadia, M. R. and Paunwala, C. N. (2018) 'Analysis of SVM kernels for content based image retrieval system', 2017 International Conference on Energy, Communication, Data Analytics and Soft Computing, ICECDS 2017. IEEE, pp. 1409-1414. doi: 10.1109/ICECDS.2017.8389676.

[10] Kashyap, N. and Singh, D. K. (2017) 'Color histogram based image retrieval technique for diabetic retinopathy detection', 2017 2nd International Conference for Convergence in Technology, I2CT 2017, 2017-Janua, pp. 799-802. doi: 10.1109/I2CT.2017.8226238.

[11] Khatami, A. et al. (2017) 'A deep-structural medical image classification for a Radon-based image retrieval', Canadian Conference on Electrical and Computer Engineering, pp. 17-20. doi: 10.1109/CCECE.2017.7946756.

[12] Khatami, A. et al. (2018) 'A sequential search-space shrinking using CNN transfer learning and a Radon projection pool for medical image retrieval’, Expert Systems with Applications. Elsevier Ltd, 100, pp. 224-233. doi: 10.1016/j.eswa.2018.01.056.

[13] Kimpan, S., Maneerat, N. and Kimpan, C. (2018) 'Diabetic retinopathy image analysis using radial inverse force histograms', ICIIBMS 2017 - 2nd International Conference on Intelligent Informatics and Biomedical Sciences, 2018-Janua, pp. 266-271. doi: 10.1109/ICIIBMS.2017.8279708.

[14] Kruthika, K. R., Rajeswari and Maheshappa, H. D. (2019) 'Erratum: CBIR system using Capsule Networks and 3D CNN for Alzheimer's disease diagnosis (Informatics in Medicine Unlocked (2019) 14 (59-68), (S235291481830176X), (10.1016/j.imu.2018.12.001))', 
Informatics in Medicine Unlocked, 16(August). doi: 10.1016/j.imu.2019.100227.

[15] Lahari, M. V. and Niranjana Krupa, B. (2018) 'Intelligent Content Based X-Ray Image Retrieval using Speeded up Robust Feature Descriptors', WIECON-ECE 2017 - IEEE International WIE Conference on Electrical and Computer Engineering 2017. IEEE, (December), pp. 70-73. doi: 10.1109/WIECON-ECE.2017.8468926.

[16] Liu, M. et al. (2019) 'Joint classification and regression via deep multi-task multi-channel learning for Alzheimer's disease diagnosis', IEEE Transactions on Biomedical Engineering. IEEE, 66(5), pp. 1195-1206. doi: 10.1109/TBME.2018.2869989.

[17] Liu, X., Tizhoosh, H. R. and Kofman, J. (2016) 'GENERATING BINARY TAGS FOR FAST MEDICAL IMAGE RETRIEVAL Department of Systems Design Engineering University of Waterloo, Waterloo, ON, Canada N2L 3G1 Centre for Bioengineering and Biotechnology University of Waterloo, Waterloo, ON , Canada N2L 3G1', pp. 2872-2878.

[18] Nguyen, L. D. et al. (2018) 'Deep CNNs for microscopic image classification by exploiting transfer learning and feature concatenation', Proceedings - IEEE International Symposium on Circuits and Systems, 2018-May, pp. 3-7. doi: 10.1109/ISCAS.2018.8351550.

[19] Qayyum, A. et al. (2017) 'Medical image retrieval using deep convolutional neural network', Neurocomputing. Elsevier B.V., 266, pp. 8-20. doi: 10.1016/j.neucom.2017.05.025.

[20] Rahimzadeh, M. and Attar, A. (2020) 'A modified deep convolutional neural network for detecting COVID-19 and pneumonia from chest X-ray images based on the concatenation of Xception and ResNet50V2', Informatics in Medicine Unlocked. Elsevier Ltd, 19, p. 100360. doi: 10.1016/j.imu.2020.100360.

[21] Soundalgekar, P. et al. (2018) 'Medical Image Retrieval Using Manifold Ranking with Relevance Feedback', Proceedings - 12th IEEE International Conference on Semantic Computing, ICSC 2018, 2018-Janua, pp. 369-373. doi: 10.1109/ICSC.2018.00075.

[22] Srinivas, M. et al. (2015) 'Content based medical image retrieval using dictionary learning', Neurocomputing. Elsevier, 168, pp. 880895. doi: 10.1016/j.neucom.2015.05.036.

[23] Swati, Z. N. K. et al. (2019) 'Content-Based Brain Tumor Retrieval for MR Images Using Transfer Learning', IEEE Access. IEEE, 7(c), pp. 17809-17822. doi: 10.1109/ACCESS.2019.2892455.

[24] Tian, Z. et al. (2016) 'SAR ATR based on convolutional neural network', Journal of Radars, 5(3), pp. 320-325. doi: 10.12000/JR16037.

[25] Wijesinghe, I., Gamage, C. and Chitraranjan, C. (2019) 'Deep supervised hashing through ensemble cnn feature extraction and low-rank matrix factorization for retinal image retrieval of diabetic retinopathy', Proceedings - 2019 IEEE 19th International Conference on Bioinformatics and Bioengineering, BIBE 2019. IEEE, pp. 301-308. doi: 10.1109/BIBE.2019.00061.

[26] Xue, Z. et al. (2018) 'Gender Detection from Spine X-Ray Images Using Deep Learning', Proceedings - IEEE Symposium on ComputerBased Medical Systems. IEEE, 2018-June, pp. 54-58. doi: 10.1109/CBMS.2018.00017. 